\title{
El empoderamiento y la biotecnología en el desarrollo profesional: una posibilidad para la supervivencia de la humanidad ${ }^{1}$
}

\author{
Gina Paola Barón González², Diego Armando Rincón Caballero 3 \\ Nubia Constanza Arias Arias ${ }^{4}$
}

\begin{abstract}
Resumen
Ser profesional en la actualidad va más allá de un saber y un saber hacer, que es el discurso predominante en términos de competencias y lo que por lo general se evalúa en las pruebas masivas como el Saber Pro. Lo profesional se diferencia de lo técnico y lo tecnológico a partir de la comprensión del significado del poder como posibilidad de transformar la realidad y del empoderamiento como la facultad que permite actuar con un sentido ético que hace que garantice la calidad de vida humana. En éste sentido la biotecnología juega un papel fundamental en el desarrollo profesional, puesto que, es el espacio donde los seres humanos podemos preservar la vida usando la tecnología, las relaciones descritas son objeto de reflexión del presente artículo, a partir del desarrollo del proyecto de investigación: Contribución de los Saber Pro (ECAES) a la mejora de la calidad de la Educación Superior: Oportunidad de Formación y transformación ${ }^{5}$.

A raíz de esa relación de empoderamiento y educación biotecnológica, subyace una reflexión en torno a los procesos formativos del profesional que asume el reto de enfrentar problematicas de diversas disciplinas y, entender de una manera crítica y propositiva la relación de un liderazgo tecnológico para afrontar problematicas dando solución desde aspectos bioéticos y humanísticos, acordes a políticas de salubridad, desarrollo sostenible e implicaciones sociales que demanda la participación activa de la comunidad en beneficio de recrear ámbitos emergentes de un empoderamiento socio-cultural como esfera global que aúna los esfuerzos del profesional y la formación biotecnológica.
\end{abstract}

Palabras Clave: Bioética, Biotecnología, desarrollo profesional, empoderamiento, objeto tecnológico.

1 Recibido: 03 de marzo del 2012. Aceptado: 26 de abril del 2012.

2 Gina Paola Barón González. Investigadora en Educación y Humanidades de la Universidad Militar Nueva Granada. Integrante del Grupo GECS, Licenciada en Filosofía de la Universidad Minuto de Dios, Diploma de estudios en Docencia universitaria, correo: gina.baron@unimilitar.edu.co

3 Diego Armando Rincón Caballero. Joven Investigador Colciencias en Educación Universidad Militar Nueva Granada. Integrante del Grupo PYDES, Licenciado en Diseño Tecnológico de la Universidad pedagógica Nacional, correo: diego.rincon@unimilitar.edu.co

4 Nubia Constanza Arias Arias. Directora Maestría en Educación Universidad Militar Nueva Granada. Líder Grupo GECS, Licenciada en Psicología y Pedagogía Universidad Pedagógica Nacional , Especialista en Educación Sexual Fundación Universitaria los Libertadores, Diploma de Estudios Avanzados en Educación DEA Universidad de Oviedo, Doctora en Educación y Medio Universidad de Oviedo, Correos: nubia. arias@unimilitar.edu.co,ncarias@hotmail.com

5 Proyecto de Investigación desarrollado en el Departamento de Educación de la Universidad Militar Nueva Granada, por el Grupo GECS en el año 2011. 


\title{
Empowerment and biotechnology professional development: a possibility for the survival of mankind
}

\begin{abstract}
Professional in today goes beyond a know and a know-how, is the dominant discourse in terms of skills and what is usually measured in the massive test as "Saber Pro". Professional technical and contrast from the technological understanding of the meaning of power as possible to transform of reality and empowerment you can act with ethics sense that makes to preserve the survival of humanity. In this regard biotechnology play vital role in professional development, as is the space where humans can preserve life using the technology, the relationships described being given to this article, from project development research: Contribution to the "Saber Pro" (ECAES) to quality on Higher education: Opportunity for training and transformation.

Following the relationship of empowerment and biotechnology education lies a reflection on the professional training processes that are challenged to confront problematic in various discipline and understanding of a critical and purposeful relationship of technological leadership to address problematic solution resulting from bioethical and humanistic aspects, in line with health policies, sustainable development and social implications that demand the active participation of the community in the emerging fields of recreating a socio-cultural empowerment as global sphere that combines the efforts of professional and biotechnology training.
\end{abstract}

Key words: Empowerment, biotechnology, technological object, professional development, bioethics.

\section{Introducción}

El empoderamiento y la biotecnología en el desarrollo profesional como posibilidad para la supervivencia de la humanidad, implica la comprensión de conceptos claves como poder, empoderamiento (crespo et al., 2007) y la influencia de ellos en la educación contemporánea y la formación de profesionales. Al mismo tiempo se requiere la compresión de los conceptos de tecnología y biotecnología como recursos que los profesionales deben emplear para su desarrollo y contribución a la supervivencia de la humanidad, estos conceptos son tratados a lo largo del presente artículo, para llegar a la conclusión que el desarrollo profesional de un individuo en la actualidad tiene como factores fundamentales el empoderamiento y la biotecnología y que funcionan como procesos adaptativo y propositivo, que permite diferenciar a los profesionales exitosos, de aquellos quienes no tiene posibilidades de mantenerse en el mercado actual, ni responder a las necesidades sociales.

La problemática se centra en determinar la importancia de una conciencia y una reflexión, ligada a la visualización de la Biotecnología como profesión que está enlazada con el tratamiento de problemas y necesidades, que el hombre está empezando a enfrentar en la sociedad de las redes informáticas y en el contexto de la aldea global (Forero, $2005,35)$. En esa medida vienen anexos el análisis de aspectos conceptuales tales 
como la tecnología en su consideración epistémica a través de su impacto sociocultural (Andrade, 1996) y la bioética como insumo disciplinar que permite entender la trascendencia de la formación del sujeto, con base en principios de respeto y retribución al ámbito ecológico y ambiental desde la relación dignificante de la condición del hombre en protección de su vida.

Este artículo surge como producto del proyecto de investigación titulado: Contribución de los Saber Pro (ECAES) a la mejora de la calidad educativa: Una oportunidad de formación y transformación, teniendo como propósito evidenciar los avances del proceso prospectivo en la educación y formación superior en cuanto a la relación del desarrollo profesional, las pruebas masivas y sus limitaciones y la concepción de la disciplina en la academia más allá de lo eminentemente procedimental o cognitivo. En el desarrollo del proceso se develan componentes importantes en el posicionamiento de los profesionales en la actualidad como el poder y el empoderamiento para impulsar su quehacer, a partir de la concepción de nuevos escenarios, donde la importancia de la biotecnología devela ejes multidisciplinares (García, 2006), como parte de la responsabilidad social-cultural que afrontan la acción participativa dentro de una sociedad.

\section{Concepción del empoderamiento}

El empoderamiento como concepto ha generado múltiples dicotomías con respecto a términos como el poder y su relación histórica con la mujer, siendo protagonista en este proceso de incursión y cambio social. No obstante, en la actualidad el empoderamiento puede ser analizado desde miradas sociales, políticas, económicas, entre otras, siempre que haya una repercusión en veras de una transformación social. Sin embargo autores como Larrea (2005) citado en Crespo et al. (2007), convergen en una visión del empoderamiento en cuestiones de subjetividad social, el cual queda desprovisto del componente político-económico para incidir en un cambio desde la atribución del poder no como proceder autocrático sino como eficacia colectiva.

El concepto inclusor de Poder está ligado al desarrollo del Empoderamiento y su instancia está concebida para entender la capacidad en comprender y asumir un cambio causal en la medida que haya una relación comunitaria, colectiva asociada al alcance de un fin común. El poder según Intrac (1999) determina una acción de diversos niveles en intereses específicos bajo escalas poblacionales de orden regional, sectorial, departamental, nacional e inclusive global. Por connotaciones culturales, el poder suele asociarse a referentes autoritarios, predominancia y un estatus jerárquico que obedece a un control desde un ámbito supremo de orden institucional o político. En su convalidación con el Empoderamiento la esfera trascendente de poder conlleva a mecanismos de participación alejada de la opción gubernamental partidista y legislativa (Aldana, 2002).

Como asegura Crespo et al. (2007) el empoderamiento difiere de la persona vista como objeto y acoge la mirada en la que el sujeto tiene como propósito el actuar en su contexto, desde la toma de posturas y decisiones para afrontar su realidad partiendo de un análisis de problemas y necesidades provistas en su dimensión de interacción socio-cultural. A la luz de lo descrito por Intrac (1999) el empoderamiento viene aplicado en tres grandes áreas:

- Poder apropiado a las cualidades y atributos de emprendimiento en campos de acción con eficiencia.

- Poder en relación con las acciones e interacciones que asume un grupo social con poblaciones, instituciones y entidades. 
- Poder en finalidad a tener un auge y reconocimiento en diferentes marcos socio-culturales para apropiarlos a beneficio.

El empoderamiento a diferencia del poder está ligado de manera directa a la acción, en él la fuerza está dirigida no al sujeto en particular sino a su actuar y la repercusión que éste puede tener en los cambios sociales. La acción consolidada en un espacio social puede convertirse en estructuras simbólicas capaces de movilizar un grupo de personas en pro de una idea u objeto común. "El sujeto se convierte en agente activo como resultado de un accionar, que varía de acuerdo con cada situación concreta" (León, 1998, p. 6). Este potenciar de la acción de los sujetos, hace posible cambios culturales e imaginarios dentro de una sociedad que en su mayoría persiguen los ideales hegemónicos que le han sido impuestos desde su historia. Empoderar posibilita reconstruir concepciones y modificar la percepción sobre alguna realidad y ocupar un lugar, el lugar de la acción.

De las múltiples concepciones de empoderamiento se acogen entre otras aquella que desarrolla Batliwala citada en (León, 1997) denominado como poder sustentable que es aquel "poder en el que las relaciones entre hombres y mujeres permitan integrar lo micro y lo macro, lo privado y lo público, lo productivo y lo reproductivo, y lo local y lo global" (león, 1997, 14). Acá el poder es visto como proceso que no se compone de dos partes opuestas, como el tener o no, el ser bueno o malo, ser estático o móvil, sino como un ejercicio que implica la multiplicidad de las relaciones humanas y la contingencia que habita lo social. Es un empoderamiento que se torna significativo según su contexto, sus objetivos, las necesidades que pretende diluir, en las interacciones cotidianas de la vida con las dinámicas que se dan dentro de las relaciones de poder.

\section{Empoderamiento y educación}

La alfabetización como acto educativo fue uno de los primeros intentos de empoderamiento, desafortunadamente su planificación no llevo a una emancipación real de cada sujeto. (Stromquist, 1997). Dado que éste no era un actor que se apropiara de su historia de manera crítica y que cuestionara su condición social, lo que hacía que sus intereses no superaran el anhelo de aprender a leer y escribir para cumplir con las exigencias básicas de su entorno.

Una educación que haga posible el empoderamiento se da mediante diversas fases; éstas inician con el desarrollo de conciencia a nivel individual y luego a nivel grupal, con el fin de generar acciones colectivas de oposición a las condiciones con las cuales hay inconformidad. Una problemática que es compartida y concretizada en un grupo de personas se desvanece al perseguir conjuntamente su cambio, movilizar los esfuerzos creando dinámicas menos nocivas dentro de la sociedad hace posible, que cada una de las personas que la compongan se cuestione sobre su condición, la modifiquen y la hereden a generaciones futuras. (León, 1997).

Ya en la acción educativa concreta debe darse antes que nada una postura de emancipación de sí misma, para no reproducir los discursos que perpetúan la clasificación y división social de la sociedad. La formación de los sujetos debe estar orientada a desarrollar contenidos tales que permitan construir críticamente un cuerpo conceptual, que configure nuevas realidades sociales, nuevos mundo posibles que contengan un sin número de oportunidades para el desarrollo de la autonomía, la autoestima y la competitividad (Stromquist, 1997). Los programas académicos deben responder a la especificidad de la población, no se puede empoderar a alguien, ni emanciparlo si sus problemáticas reales y cotidianas son desconocidas y por ende ignoradas. 


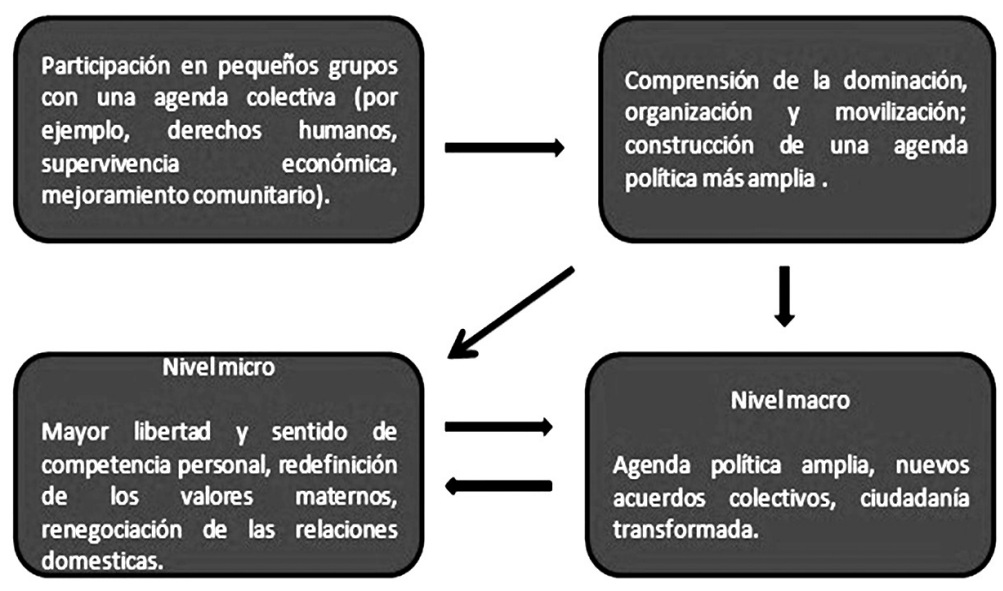

Figura 1. Cadena teórica de eventos en el proceso de empoderamiento (cc).

Emprender una educación como ésta requiere un cambio de mentalidad, un espacio de reflexión y discusión críticas que lleve a la configuración de una racionalidad para el empoderamiento, en la que hombres y mujeres discutan desde su experiencia de vida, las necesidades que han surgido y la posibilidad de cambio de roles para lograr un desarrollo más humano y equitativo que beneficie su entorno social.

Una racionalidad para el empoderamiento, hace que el ejercicio educativo tome un modelo de aprendizaje que involucre la vida diaria de quienes participan en él. Ejercer el dialogo abierto a las experiencias de género permite que al relatar la identidad y necesidades de cada sujeto, se vaya convalidando la de las otras personas que pertenecen al grupo. Un reconocimiento público de sí mismo, evidencia la forma en la que la vida de cada quien está ligada a la de los que lo rodean, y hace evidente cómo sus actos permiten trasformar sus identidades particulares y las de la comunidad o cultura a la que pertenecen. (Stromquist, 1997)

En el caso concreto de una educación biotecnológica el diálogo entre géneros es indispensable para poder configurar una sola idea sobre el cuidado de la vida y desde allí hacer un uso adecuado de lo tecnológico. Históricamente el hombre ha heredado en su formación y desempeño laboral el compromiso por responder a la idea moderna de desarrollo en la que los recursos naturales son maltratados. A diferencia de la mujer en su rol de madre, el cuidado de la vida y los recursos le permiten entender frente a la idea de desarrollo otra forma de intervenir en los procesos vitales de los seres humanos y de la naturaleza. Lo que es más acorde a responden las exigencias sociales y del medio ambiente que reclama hoy el mundo.

Con esto no quiere estigmatizarse el trabajo del hombre, sino hacerse un llamado a reconocer las identidades heredadas que contribuyen o estropean el ejercicio de la configuración de un mundo más equilibrado en temas de bioética, de humanidad, de políticas de salubridad, y de desarrollo sostenible entre otros. La visión del hombre puede complementarse con la mirada del cuidado de la vida que se le ha asignado culturalmente a la mujer y emprender una participación activa de dicho cuidado sin importar pertenecer a género masculino.

De igual forma, en diálogo con lo masculino, la mujer puede tener un conocimiento y manejo de la parte operativa y de los desarrollos funcionales que por el mismo machismo se han concentrado históricamente en manos del hombre. Así, es posible generar una actitud de alianza y respeto con el otro (Kabeer, 1997), pues 
entiendo y valoro su conocimiento y percepción de mundo, como formas validas que pueden dar solución o tratamiento diferente a aspectos que antes no han podido solucionarse.

Lo importante de este ejercicio es generar un lugar abierto para discutir frente a la formación académica y el posterior desempeño profesional. Y la manera en que se deben suplir las necesidades de género, y cómo poner en diálogo las apuestas que desde las diferentes partes para llegar a un concepción y uso adecuado de o biotecnológico.

Por todo lo anterior, es necesario postular de manera clara los retos más relevantes en la formación e implementación de lo biotecnológico y aunque sean difíciles se debe pensar en que son procesos lentos, dado que como acto previo debe haber concientización para modificar la conducta. Hacerse consiente es revisar los proceso personales de identidad de cada sujeto y a su vez la carga heredada desde su cultura de origen, para así poder, hablar con claridad las diferencias o semejanzas con otros sujetos o culturas que también los configuran en este mundo globalizado.

Esta tarea de la educación acompañar ese cambio acción pues debe ser dialogada y reconfigurada a partir de la confrontación entre ideas previas y posteriores a ella. La educación debe mostrar a los sujetos que el diálogo sobre la necesidad de cambios sociales, se vuelve significativo sólo si se concretiza en movilización fuera de la institución educativa, si se crean grupos específicos que persigan un interés particular, lejos de agentes que controlen su actuar. Es en este tipo de espacios donde se logra crear conciencia insurgente (Stromquist, 1997, 75), una conciencia que retumbe en el pensamiento de quienes dominan, pero no con manifestaciones de masas sino con actos cotidianos que reevalúan la lógica común del deber ser.

Para esto, educar en el empoderamiento es crear una mentalidad de autoevalua- ción constante que siempre esté indagando el propio quehacer y que permita sin temor alguno modificar la acción si ésta no responde a las necesidades equitativas y beneficiosas para la comunidad en general.

\section{La percepción actual de la tecnología}

La concepción Tecnológica presenta en la actualidad una serie de dicotomías que van relacionadas con la sofisticación y la innovación, desde el punto de vista de la educación por competencias que se ha venido trabajando desde los planteamientos generales de la UNESCO. Sin embargo, el tomar este enfoque, devela un arma de doble filo que lleva a la sociedad a interpretar el concepto de tecnología como el facto, es decir el prototipo más avanzado o los productos, bienes y servicios que abanderan los procesos industriales, comerciales y productivos en los contextos de países potencia y en otros que están en marcha hacia este camino de crecimiento.

Por lo tanto se hace necesario recalcar que la Tecnología en su estatuto epistémico no corresponde únicamente a dicha percepción objetual, sino que como enfatiza García (2006) es una actividad humana que está concebida para la transformación social y del entorno, llevando al hombre a satisfacer una serie de necesidades y problemáticas circundantes en su interacción sociocultural. Lo cual hace pensar que su relación y asociación con artefactos innovadores está mediada por los procesos y conocimientos que confronta el hombre para dar respuesta mediática a través de los objetos bien sea de uso cotidiano o en un orden de producción masiva e industrial.

Recientemente muchos autores y estudios han volcado su interés a reflexiones que den cabida a entender la incursión tecnológica desde el marco educativo, como evento formador y alfabetizador del ser en su papel transformador de los recursos a 
partir de la tecnología como epicentro y marco teórico. Así pues Andrade (1996) habla de una alfabetización del ser, para determinar culturalmente la importancia de la tecnología en la construcción de sujeto y comunidad.

Tomando entonces la consideración de la acción deliberada como recurso e insumo para llegar al "Objeto Tecnológico" (Buch, 1999), se puede enunciar que el saber tecnológico no es únicamente la consecución de una idea para ser materializada, también obedece a referentes creativos y de diseño que otorgan a la tecnología un campo de aprendizaje basado en discusiones, debates, proyectos y problemas que permiten la relación del hombre con diversos agentes abstractos y a la vez físicos. Obedeciendo a coyunturas y estructuras que demarcan civilizaciones en una temporalidad que denota la existencia de sociedades, en una carrera por entender todo aquello que les rodea. En ese diálogo se puede analizar entonces la estrecha relación de la tecnología con la ciencia y la técnica (García, 2006), donde lo deductivo y el marco disciplinar interactúan para dar respuestas constantes a leyes y aspectos que certifican "paradigmas" (Kuhn, 1971), en miras de convalidación de conocimientos sobre los cuales en la actualidad la tecnología posee una relación con la ciencia en una resultante de innovación según la UNESCO.

La principal analogía es que el hombre como especie al depender de lo natural bajo la acción de la transformación en el curso de la evolución, ha pasado a ser parte del artificio (González, 2008, 2), aspecto que lleva al sujeto a crear un reflejo quizá subjetivista de la realidad y dependiente al uso de factos enmarcados en distintas líneas donde se vislumbra la acción tecnológica. Ejemplo de lo dicho puede asociarse a la importancia de los estudios científicos apoyados en los objetos tecnológicos y en las teorías relacionadas, donde la psicología, la medicina, la ingeniería entre otras hoy en día hacen uso del saber tecnológico para producir objetos que velen por la calidad y prolongación de vida, un hecho en concordancia con las cosmovisiones generadas por la era globalizada en la cual nos encontramos hoy en día.

\section{La biotecnología y el contexto nacional}

La biotecnología surge como un proceso mediático, debido a las inquietudes de las sociedades industriales y en vía de desarrollo, en tanto cobra importancia la perspectiva basada en la preocupación y el entendimiento de los referentes de seguridad, salud y salubridad, para la humanidad y el medio circundante, incluyendo, el sector rural y el urbanístico en cada una de las dimensiones de interacción social y cultural (Jaffé, 1992). De acuerdo a esta premisa, la biotecnología se postula como una tecnología provista de la intervención pública, al contemplar medidas para contrarrestar el impacto de las nuevas tecnologías y, el desconocimiento acerca de los riesgos existentes en cuanto al uso de productos, bienes y servicios y estructuras de masificación que puedan afectar el equilibrio natural.

Jaffé (1992) incluye concisamente en dicha perspectiva de preocupación, el componente de la investigación y el desarrollo (I+D) para establecer resultados que contrarreste el impacto negativo a nivel ambiental, no obstante, en la actualidad se integra el concepto de innovación, que si bien es parte del desarrollo competitivo, converge desde el punto científico y tecnológico en diversos esfuerzos efectuados por los países globalizados, para contrarrestar los efectos de la industrialización y el manejo indebido de recursos naturales.

La biotecnología no solamente hace mención al factor de bioseguridad, también devela un interés particular por los efectos de orden socio-demográfico, económicos y políticos, siendo cada uno de estos aspectos, relevantes para atender la complejidad del impacto biotecnológico 
en épocas contemporáneas. Actualmente, se trata de implementar distintas normas y concesos a favor del uso adecuado de las tecnologías industriales, agrarias, comunicativas, entre otras, sin demeritar el avance del hombre para sobreponerse a las adversidades, en términos de adaptación con el entorno y el acervo factual que subyace frente al mejoramiento de la calidad de vida.

A nivel de países desarrollados, en el presente siglo, se ha tomado mayor conciencia hacia la responsabilidad tecnológica y la relevancia sobre una serie de políticas educativas y normativas esenciales para establecer un equilibrio del uso de tecnologías, sobre todo en el campo ambiental. Aunque, según lo afirmado por Jaffé (1992), hay mayor peligro ambiental en países subdesarrollados en los que las plantaciones y los cultivos pueden tratarse de manera incorrecta (Transgénicamente) en consecuencia a la falta de políticas y de masificación de la producción, afectando el equilibrio natural y los impactos de segundo orden; alteraciones y deficiencias relativas a enfermedades posteriores en los consumidores o sus descendientes.

En ese orden de ideas, Latinoamérica ha sido uno de los sectores con países en carrera de desarrollo económico, que ha sido impactada por la intervención de las problemáticas ambientales, ecológicas y de salubridad. Los estudios frente a la toma de conciencia y reflexión sobre las implicaciones de la biotecnología en Latinoamérica datan de finales del siglo $X X$, al compartir, particularmente en este estudio, lo expuesto por Quintero y Solórzano (1989), quienes hablan en una trascendencia temporal de cómo la inversión extranjera y los tratados para el movimiento mercantil, pudiesen afectar el presente siglo, a raíz de una biotecnología caracterizada por un avance tecnológico moderado, llevado a cabo por las transnacionales y unos tradicionales procesos operativos que se realizan internamente por mano de obra a bajo costo y la "transferencia tecnológica" (Quintero y Solórzano, 1989, p.188), consecuente a la abundancia en la materia prima de estos países, salvo los procesos de emancipación tecnológica abanderados por Brasil, argentina y chile.

La biotecnología en el sector latinoamericano, por lo menos en la parte aplicada de su concepción, ha sido producto de una indirecta llegada de elaboraciones científicas, tecnológicas y comunicativas (Quintero y Solórzano, 1989), que se han encargado de propiciar, en efecto, una preocupación resonante en los sectores políticos y comerciales para la toma de medidas benefactoras a la preservación de los recursos ambientales y el patrimonio natural, característico de cada nacionalidad. Las oportunidades para considerar la protección de estos bienes, aún son escasas, y se basan en planteamientos que a veces resultan insuficientes, considerando la magnitud del fenómeno industrial adelantado en vísperas del duodécimo año del presente siglo.

Particularmente, en el contexto colombiano, han crecidos los estudios e investigaciones referentes al campo biotecnológico. Sin duda alguna, la preocupación es latente y cada unos de los sectores, industriales, comerciales, agrarios y humanísticos han develado las problemáticas centrales del uso indebido de recursos, procesos y estrategias que atenten contra el patrimonio natural. Esta afirmación, del aumento de investigaciones biotecnológicas en Colombia, se basa en el estudio desarrollado por Orozco y Olaya (2005), acerca del análisis de investigaciones adelantadas para contrastar el impacto de las actividades administrativas y científicas que sopesan el tratamiento biotecnológico, acorde a procesos políticos y gestiones para el cambio en diversos sectores rurales y urbanos del país.

Una muestra importante de la aplicación biotecnológica en Colombia, está enmarcada en el sector agrícola y biológico, debido a la diversidad de especies y el cultivo de múltiples frutas, verduras 
y legumbres con tratamiento tecnológico de ingeniería para el cultivo, protección, recolección y contención de los productos (Arias, 1994). Colombia cuenta con observatorios biotecnológicos, entidades legisladoras; veedoras del cumplimento de normas y estándares, e institutos para el tratamiento tecnológico de los alimentos. Por otra parte, según estudios de Orozco y Olaya (2005), cuenta con 56 grupo de investigación reconocidos ante Colciencias que trabajan la parte biotecnológica $y$, a su vez, en cada grupo se encuentran alrededor de 162 Doctores y 248 Magíster, liderando los proyectos que incurren en líneas de investigación con énfasis en la temática biotecnológica. Cabe anotar además, retomando el estudio de Orozco y Olaya (2005), la siguiente información relacionada con los integrantes de los grupos de investigación que trabajan la biotecnología en Colombia de acuerdo al género y el nivel de estudios:

Tabla 1. Relación de integrantes de acuerdo al género y nivel de estudio, pertenecientes a grupos de investigación asociados con la Biotecnología en el 2002

\begin{tabular}{|l|r|r|r|}
\hline Nivel de Estudio & Femenino & Masculino & Total \\
\hline Doctorado & 65 & 118 & 183 \\
\hline Maestría & 226 & 200 & 426 \\
\hline Especialización & 42 & 46 & 88 \\
\hline
\end{tabular}

\section{La biotecnología, un campo interdisciplinario}

La Biotecnología no es un eje temático singular, está conformada de varias disciplina, que a la par, se alimentan de los desarrollos descendientes de la práctica epistémica y metodológica convocada en el saber biotecnológico. Hay que aclarar; la interdisciplinariedad como afirma $\mathrm{Mu}$ ñoz (2001), no es la conjunción de partes temáticas de diversas disciplinas, se trata más bien, de un proceso depurativo de cuerpos teóricos convocados por lo objetivos comunes de las disciplinas implicadas, bajo esa salvedad, la biotecnología es interdisciplinar, toda vez la coherencia de las finalidades y objetivos disciplinarios, establezcan nuevos enfoques; flexibles, si se quiere, para abordar problemáticas complejas en relación a las tecnologías y el uso adecuado bajo un contexto definido.

También puede referirse a la Biotecnología, como un espacio multidisciplinar que hace extensión a un acervo de conocimientos, cuya estructuración epistemológica no deja la identidad de cada disciplina (Muñoz, 2001), más aún, convoca relaciones fundentes en procesos y métodos capaces de afrontar aspectos como las políticas globales, impacto industrial en el medio ambiente, las nuevas tecnologías y el componente filosófico y humanístico que cruza transversalmente la condición biotecnológica.

La biología; molecular y química, la ingeniería; con derivaciones: industriales, ambientales y genéticas (Muñoz, 2001), además del derecho y la política, son algunas de las disciplinas que intervienen en el análisis de los problemas biotecnológicos, sin duda alguna, un ejemplo de interdisciplinariedad y multidisciplinariedad yace en la biotecnología con vinculación de procesos tradicionales, como es el caso de la transformación y procesamiento de alimentos, así como las llamadas tecnologías emergentes, caracterizadas por la manipulación genética con impacto en el ser humano y el ambiente.

Avalos (1990) complementa el aspecto interdisciplinar de la biotecnología, con la aplicación de esta tecnología emergente. Asimismo, son varias las aplicaciones de este saber interdisciplinario: la agricultura, la producción de alimentos, la salud, el refinamiento de energías, la industria farmacéutica, química avanzada y uno de los más importantes; la preservación del medio ambiente. Aunque, estudios más recientes, revelan que la principal fuente de aplicación biotecnológica reside en el tratamiento de alimentos, incentivando los procesos de producción, el aceleramiento de la cosecha y el manejo de la calidad y conservación de estos víveres. 
Los campos de producción más significantes en cuanto a aplicaciones biotecnológicas son, por un lado, el de los alimentos que cuenta con: la producción de proteínas y la sustitución de maneras convencionales para producir el azúcar; los biocarburos y productos químicos, destinados al uso en el tratamiento de combustibles y fermentaciones específicas respectivamente (Avalos (1990). Conjuntamente, estos campos fructíferos dependen de la producción de biomasa, concluyendo que el campo agrario y alimenticio son los más destacados en este tipo de procesos biotecnológicos.

\section{Empoderamiento y biotecnología desde la relación bioética}

El interés creciente que ha adquirido la ciencia y la tecnología en un ámbito publico fuera de los lindes académicos, ha generado una serie de expectativas en cuanto los intereses que conllevan a preguntase y cuestionar la repercusión e impacto que puede tener los desarrollos tecnológicos vistos desde esta perspectiva, a la luz de la incidencia de los objetos tecnológicos. Es así como Carullo (2002) argumenta este hecho bajo la relación de la tecnología con otras áreas, en la cuales la población en general obtiene una participación activa, sin demeritar el aspecto socio-económico e incidir peyorativamente en aseveraciones elitistas; En países desarrollados y los que están en esa marcha.

Teniendo este marco contextual Durant \& Geoffrey citados en Carullo (2002), distinguieron una serie de beneficios que dan cabida a reflexionar en el análisis de la compresión de los hechos científicos y tecnológicos en distintos niveles de la población. Estos Ítems son expuestos a continuación:

a) Beneficios para la ciencia: Desde la permisividad y aproximación hacia las investigaciones generadas bajo el lente científico, logrando en cierta medida la concentración de diversas miradas que pueden contribuir desde el aporte intelectual, físico y monetario.

b) Beneficios para las economías nacionales: Aquellos países que abanderan e incentivan la tecnología y su PIB demanda un nivel considerable de inversión hacia la educación para la innovación, encuentran mayor competitividad en el sector productivo.

c) Beneficios para la influencia y el poder nacional: Desde lo enunciado en el anterior tópico, en el sentido de una pertenencia nacional y una pertinencia para la competitividad con estamentos, empresas y organizaciones internacionales.

d) Beneficios para los individuos: Cada persona que obtiene información y mayor participación en las investigaciones de orden científico y tecnológico, pueden acceder a mayores oportunidades, equivalente a mejores condiciones de vida en un desenvolvimiento eficiente en la sociedad.

e) Beneficios para el gobierno democrático y para la sociedad: incentiva la imparcialidad desde la información objetiva, puesta a consideración por diversos estamentos que dan como resultado el componente crítico y reflexivo ante fenómenos y estructuras que pueden ser puestas en tela de juicio desde aportes validos a partir de la construcción social.

f) Beneficios intelectuales, estéticos y morales: El saber, el conocimiento como insumo juega un papel importante como agente motivador, además de inculcar respeto por la propiedad intelectual y la estimulación hacia la investigación como recurso próximo hacia la adquisición de saberes en distintos contextos.

Si se analiza a profundidad cada uno de los anteriores aspectos, se puede empezar a visualizar la relación que posee la tecnología con el empoderamiento; toda vez que prevalezca el aspecto denotado en este apartado, el cual hace mención al interés de los conceptos científicos y tecnológicos por parte de grupos y comu- 
nidades que ven en dichos ejes la posibilidad de una transformación social, como un mecanismo de acción participativa, donde la sinergia conlleva a la ruptura de paradigmas y al establecimiento de nuevas condiciones de interacción; En pro del beneficio colectivo, sin demeritar el hecho de la incidencia del poder y el condicionamiento bajo normativas y jurisdicciones que van desde lo político hasta limitantes de orden económico.

La bioética como principio y disciplina que ha venido trabajando ese elemento de incidencia de los aspectos natural-artificial en la psique humana, permite entender con mayor precisión la repercusión de las variaciones ambientales, científicas e inclusive tecnológicas en la vida del ser y en la conciencia misma de la importancia del análisis de las conductas humanas como resultado del impacto del balance natural y artificial. Extrapolada esta situación dual a un lenguaje matemático, se propondría analógicamente una regla de tres en la cual el factor incluyente seria el científico-tecnológico, buscando hallar la filosofía y "sapiencial" (García, 2006) desde los valores y principios formativos del ser humano.

La Bioética y su vinculación al campo académico, científico y tecnológico, compromete en cierta parte una interpretación y comprensión dirigida desde espacios como la Universidad y las instituciones de Educación Superior, en los cuales la formación del estudiante propenda por una proyección del desarrollo humano sostenible (Rascio, 2007), siendo ésta, prioridad de todo marco formativo profesional en veras de una igualdad y preservación de la vida en constancia con la realidad de la sociedad del conocimiento en la que está inmerso el hombre actualmente.

Con lo mencionado en este apartado se quiere resaltar la importancia de vincular una concepción de Bioética a los aportes que genera la tecnología en la profesión creciente de la Biotecnología, la cual refiere al uso de organismos vivos y sus partes para el aprovechamiento desde un fin específico, teniendo en cuenta además su implementación en aspectos tradicionales en cultivos, la domesticación de animales y el uso de plantas en procesos curativos (Muñoz, 2007). A la vez, su apreciación de forma moderna, basada en estudios hechos por H. Boyer y S. Cohen en 1973 citados en Muñoz (2007), dando paso a la incursión genética como forma de adquirir beneficios desde el estudio de la biología molecular. Por tanto, la controversia sobre dicho procesos ha generado múltiples miradas y opiniones al respecto, donde quizá la bioética hoy en día juega su rol de mediador respetando los derechos y límites que este tipo de manipulación genética pueda tener en la sociedad y la concepción de ser humano.

En el congreso auspiciado por la Organización de desarrollo industrial de naciones unidas [ONUDI] citado en Carullo (2002), en el 2001 se dieron como premisas importantes la relevancia y crecimiento de la biotecnología con respecto a su enfoque multidisciplinar, en dónde además de intervenir las ciencias médicas y humanas, se reúnen disciplinas económicas, políticas y empresariales que en resumidas llevan a reflexionar de manera prospectiva sobre la importancias de la profesión, en consideración de los avances tecnológicos y los cuestionamientos que realiza la gente sobre la moralidad con base en la práctica y técnica asociada a métodos microbiológicos, impulsados cada vez más como alternativas a muchos dilemas que el hombre espera abordar en el mundo globalizado (Rascio, 2007).

\section{Un empoderamiento biotecnológico}

El empoderamiento a razón de lo argumentado a lo largo de la investigación conlleva a una participación en el sentido de informar al público sobre el significado y consolidación de la biotecnología, no únicamente como manipulación indebida 


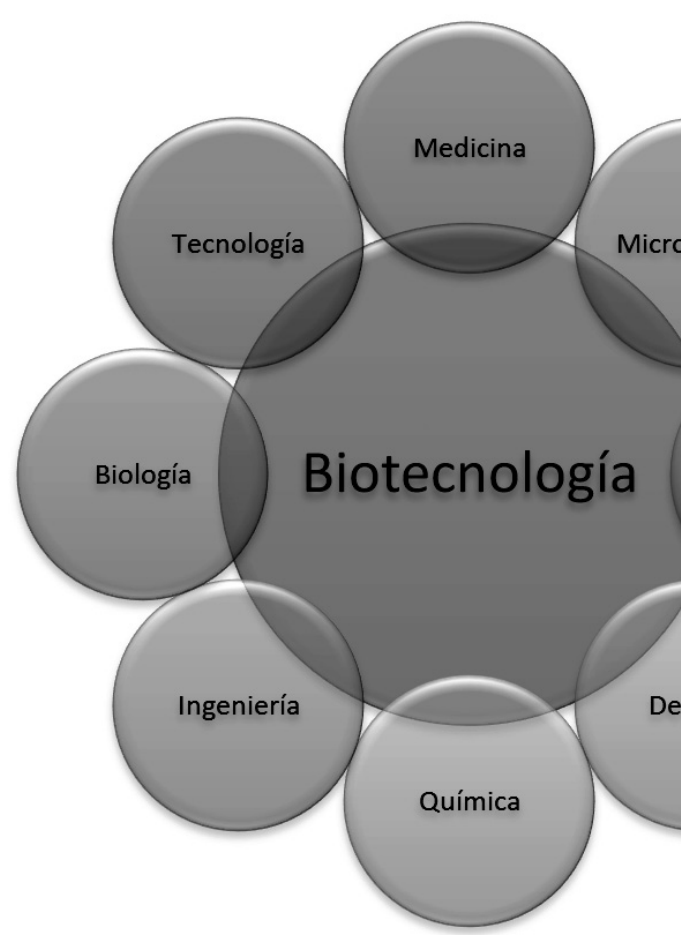

de procesos genéticos, sino desde la perspectiva en la cual el crecimiento demográfico y el cambio socio-cultural van a un ritmo acelerado que debe ser contemplado en el análisis de la preservación de la vida y en las medidas que se optan para establecer una calidad de vida desde la integridad, el respeto y la responsabilidad del hombre conforme transforma su entorno natural para desarrollar artificios en pro de su beneficio.

En otra instancia el empoderamiento no sólo se ejerce desde la participación e información del colectivo, en una mirada particular, los profesionales y los organismos educativos deberán orientar la profesionalización de los educandos relacionados al campo biotecnológico en el rol que asumen para la contribución a una práctica científica y tecnológica, con base en los principios de valores que ofrece la Bioética para una aplicación de conceptos sopesados sobre una conciencia y causalidad por la que se ejerce el derecho a la construcción de sujeto, en
Figura 2. Disciplinas relacionadas con la Biotecnología.

cuanto se fundamente la inculcación de principios en un plano racionaldialógico que ofrece la "deontología profesional" (Ferrero, 2007).

El profesional prospectivo en Biotecnología debe obtener una serie de competencias que atiendan a necesidades que constantemente están surgiendo a causa del incremento de los factores de centralización y problemáticas que radican en pobreza, hambruna, enfermedades provocadas por nuevas mutaciones de virus y productos sustentados para mejorar la calidad de vida.

Así, bajo esta mirada la concepción de desarrollo debe orientarse a la participación activa y planificada de las comunidades que vayan a ser trasformadas con una decisión de corte biotecnológico. Debe posibilitarse según Young (1997) una consulta activa y constante que permita hacer evidente la heterogeneidad de los sujetos y las necesidades que emanan de ellos. La constancia en la consulta a la comunidad hace el proceso aterrizado, no se puede pensar que todas las mujeres poseen las mismas necesidades o prototipos de vida, caso que también aplica para los hombres y sus variedades.

La consulta debe estar acompañada de información clara y precisa para que se medie entre derechos y deberes que tiene la comunidad dentro de un proceso biotecnológico. En el caso de procesos de cambio a las condiciones de las mujeres, la consulta ha generado resultados valiosos que pueden implementarse en 
el campo del empoderamiento en la biotecnología.

Estas reflexiones sugieren que si los planificadores quieren enfrentar las necesidades (...), primero deberán tener la capacidad de promover la identificación de estas necesidades; luego, siempre que sea posible en la consulta (...), deberán evaluar su capacidad para satisfacerlas $y$, nuevamente e la consulta dar prioridad a aquellas que son susceptibles de una solución política o a través da la planeación. (...) La consulta no puede restringirse sólo a la planeación; el dejar de lado una continua retroalimentación en todas las etapas del proyecto con mujeres plantea un problema, porque el proceso de consulta, que debe ser continuo, se limita exclusivamente a la etapa de planeación. No obstante, es en el curso de la implementación como parte del proceso que la necesidad de cambios lajustes se torna muy clara. (Young, 1997, p. 101)

Cuando el profesional se hace consiente de este tipo de planeación y la evaluación constante que debe haber sobre ella, el empoderamiento va configurándose, puesto que la mirada de la comunidad se convierte en insumos para la innovación. Y si la consulta y sus constantes ajustes como dice Young (1997) dependen de lo que las voces de la comunidad emiten, la mirada crítica se hace presente, pues se evalúa el bienestar particular y general de los participantes en contraste de una propuesta biotecnológica que debe darse desde dentro y no como ente externo autoritario y conocedor absoluto de lo que debe hacerse.

Sin embargo como trasfondo a este fenómeno, el profesional que participa en el campo biotecnológico, deberá lidiar con detractores que a causa de información fragmenta y antecedentes investigativos que cuestionan la naturalidad; caso de la conclusión de la secuencia del borrador del genoma humano en el 2001 (Muñoz, 2007, 35), pueden llegar a transformar el empoderamiento de ciertos sectores sociales en su contra, en los que movimientos activistas y eco-ambientales vean en estos académicos una posible amenaza a las leyes de naturalidad y conformación genéticas de las especies en el mundo.

Otros autores hablan de concepto de la "Nueva Biotecnología" (Muñoz, 2001, 19) implicando la manipulación genética en la que los animales en domesticación obtienen mayor producción, las plantas serian inmunes a bacterias en la que se privilegia la reconstrucción de espacios ambientales, abatidos por la mano del hombre. El profesional implicado en el campo de la innovación divergente desde ideas creativas para buscar soluciones, obtendrá responsabilidades en las que no podrá alejarse de los ejes reflexivos que apelan a los derechos y normativas que protege el libre desarrollo de los hombres y las especies en concordancia con las tecnologías crecientes para el refinamiento de las técnicas y los procesos en el análisis de organismos y micro estructuras destinadas a la producción de soluciones e iniciativas para la prolongación sana de la vida.

Los profesionales que desarrollan este tipo de actitud estarán ejerciendo lo que se conoce como empoderamiento desde dentro. Realizan una reelección y evaluación constante de las condiciones en las que se encuentra su área de actuación, "a fin de poner de relieve la base socialmente construida y socialmente compartida de problemas que a primera vista son individuales" (Kabeer, 1997, p. 135)

Cuando se rastrea este tipo de procesos se hace evidente la necesidad de un apoyo económico que propicie autonomía para ejercer las estrategias propuestas, para ello el cuerpo profesional del campo de la biotecnología debe acudir al dinero de OND o del estado, haciendo uso de sus habilidades cognitivas y verbales para mostrar la importancia de sus proyectos 


\begin{tabular}{l}
\hline Suelo y Agua \\
\hline -Teoría del \\
humus \\
-Miderales \\
-Suelos \\
- suministro de \\
Agua \\
- Fertilizantes \\
- Cultivos \\
\hline
\end{tabular}

\begin{tabular}{l}
\hline Ciencia Vegetal \\
\hline -Ecología \\
- Nutrición \\
- Genética de \\
especies \\
- Patología de \\
vegetales \\
- Frutosy \\
cosechas
\end{tabular}

\begin{tabular}{l}
\hline Ciencia Animal \\
\hline - Fisiología \\
- Genética \\
-Ecología \\
- Etologí \\
- Ganado y \\
crianza \\
- Vanuas \\
- Enfermedades \\
\hline
\end{tabular}
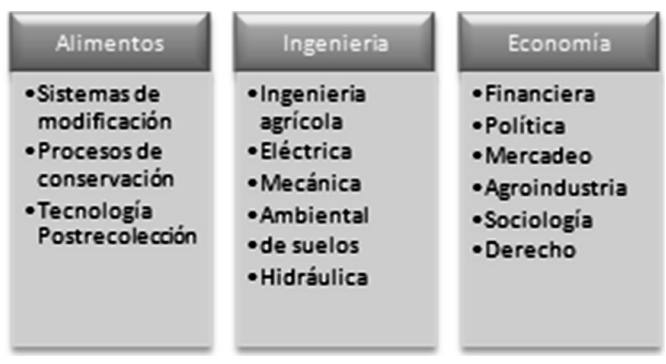

Figura 3. Relación de la biotecnología en distintas áreas disciplinares. Modificado de Muñoz (2001), (cc)

y el beneficio que causa en la sociedad. (Kabeer, 1997). Al lograr financiaciones de este tipo, se va generando de manera paulatina una conciencia hacia el cambio, trasformando la conciencia colectiva y los grandes agentes que consolidan las estructuras hegemónicas, filtrando nuevos intereses acorde a una población que antes estaba invisibilizada.

Haciendo mayor énfasis en lo dicho anteriormente, se hace una cavilación esquemática que propicie una descripción de los aspectos y áreas en las cuales esta profesionalización en auge tendría impacto, generando un cambio social y una expectativa resonante en aquellos eruditos, críticos e investigadores de diversas disciplinas:

La connotación social y el subsecuente empoderamiento que puede adquirir la Biotecnología reluce en el estudio del impacto dialectico de su afín, en relación a los cambios y transiciones que puede generar en los países que le incentiven bajo una perspectiva que involucra la inversión, los recursos, el pensamiento innovador y un contexto desinhibido de prejuicios arraigados a los cuestionamientos que implica el tratamiento genético, a través de las críticas basadas en la moralidad y la concepción del ser humano.

Tabla 2. Relación del Desempeño profesional con áreas y procesos biotecnológicos

\begin{tabular}{|l|l|}
\hline \multicolumn{1}{|c|}{ Área de dominio } & \multicolumn{1}{c|}{ Acciones del profesional } \\
\hline Energía y Minería & $\begin{array}{l}\text { El profesional podrá generar estudios relacionados con la generación de combustible alterno y } \\
\text { purificación del mismo. Además del tratamiento de diversos recursos como el agua y el sol para } \\
\text { emitir fuentes de energía renovable. }\end{array}$ \\
\hline Ingeniería e Industria & $\begin{array}{l}\text { El profesional producirá elementos tales como enzimas, vitaminas, ácidos y factos para proveer } \\
\text { a los sectores industriales. }\end{array}$ \\
\hline Procesos ambientales & $\begin{array}{l}\text { El profesional analizará el tratamiento de desechos tóxicos, basuras, radioactivos. Por otro lado } \\
\text { centrará especial atención al proceso de aguas residuales y la reducción de efectos contaminantes } \\
\text { en el ambiente. }\end{array}$ \\
\hline Agricultura & $\begin{array}{l}\text { El profesional producirá elementos como abono, fertilizante, medicamentos basados en plantas } \\
\text { alternativas, establecimiento de cultivos a prueba de plagas y reforestación de bosques talados. }\end{array}$ \\
\hline Tratamiento de alimentos & $\begin{array}{l}\text { El profesional realizará aportes para llevar a cabo alimentos transgénicos, proteínas, conservantes } \\
\text { y aditivos con mayor calidad para su consumo. }\end{array}$ \\
\hline salud & $\begin{array}{l}\text { El profesional producirá medicamentos, vacunas, tratamiento de bacterias, antibióticos y estudios } \\
\text { genéticos para evitar o curar enfermedades terminales o cancerígenas. }\end{array}$ \\
\hline biotecnología & $\begin{array}{l}\text { El profesional utilizará la microelectrónica, biomecánica, física y la nanotecnología para desarrollar } \\
\text { diminutos dispositivos que permitan controlar internamente y de forma inteligente enfermedades } \\
\text { del hombre. }\end{array}$ \\
\hline
\end{tabular}


A continuación se presentan en análisis a lo descrito sobre la profesionalización biotecnológica, un cuadro ilustrativo en áreas de desempeño con respecto al impacto que se prevé a partir de su consideración en escenarios de formación superior:

Las tecnologías contemporáneas (Andrade, 1996), ofrecen y seguirán brindando nuevas alternativas sobre las cuales numerosas operaciones y practicas se irán reformando en relación a empresas y grupos con base tecnológica para generar insumos mucho más sofisticados, acordes a procesos de miniaturización, estética y funcionalidad. En esa medida el profesional en Biotecnología tendrá a la mano el conocimiento y las herramientas para desarrollar nuevos procesos y acciones que obtengan gran impacto en la comunidad. Los "Proyectos Tecnológicos" (Buch, 2009), serán el pretexto de múltiples grupos de investigación reunidos con el fin de llegar a plantear soluciones radicales a partir de ideas e invenciones sucintas de la concientización de los impactos socioambientales generados en la naturaleza.

La denominada "tecnología de los fines" (Buch, 2009) que apela a la caracterización de las innovaciones enmarcadas sobre ámbitos como los alimentos, medios de transporte, tecnología militar y médica, han llevado a considerar nuevos espacios en los cuales la Biotecnología abarca un campo de interacción para llevar a cabo una finalidad educativa y transformadora; procedente de las problemáticas que se prevén estarán presentes en la era globalizada. Duque $(2010,33)$ realiza una descripción de estos campos de acuerdo a una serie de aplicaciones en sectores específicos:
Esta clasificación es una de tantas que se han llegado a realizar con respecto a la aplicación y los campos de actuación del futuro profesional en biotecnología. En esa mirada analítica de los comicios laborales, es necesario también manifestar la trascendencia y acogida de su concepción en diversos países, al menos en un rastreo de miradas y posturas que tienen los países latinoamericanos con respecto a los avances realizados en Estados unidos y Europa, quienes han abanderado estrategias de gran bloque (Muños, 2001, p.51). Desde la investigación realizada por Corullo (2002) se hace una reflexión sobre estudios específicos de algunos estudios específicos, por ejemplo en Brasil el tratamiento de la Soja, en Chile de la minería, en Colombia en tratado de Cartagena entre otros países que también conciben la biotecnología como optativa profesional de educación superior.

Estas descripciones sólo contemplan a grandes rasgos la incidencia de la Biotecnología en ciertos países latinoamericanos, sin ser los únicos y tampoco los más avanzados en cuestión de aplicación de tecnologías e investigaciones al respecto, si permite vislumbrar un panorama en el cual la incertidumbre y las posturas de diferentes sectores sociales, políticos y económicos, dan a relucir la concepción biotecnológica como algo ineludible y que a la larga se manifiesta en estudios emergentes con respecto a reflexiones sobre cómo desde la cultura global y de la información el hombre contempla su interacción con el medio circundante sin entrar en detrimento de sus componentes y establecer un equilibrio ambiental desde el aprovechamiento de los recursos en pro de mejorar la calidad de vida.

Tabla 3. Propuesta de aplicación biotecnológica, Modificada de Duque (2010)

\begin{tabular}{|l|l|}
\hline \multicolumn{1}{|c|}{ Tipo } & \multicolumn{1}{c|}{ Aplicación } \\
\hline Blanca & $\begin{array}{l}\text { Es aplicada a contextos Industriales en los cuales se acrecienta el estudio para regular y gestionar } \\
\text { procesos de mejor calidad. }\end{array}$ \\
\hline Roja & $\begin{array}{l}\text { Está referida a ámbito de la salud y su incursión está mediada por estudios que determinen observaciones, } \\
\text { diagnósticos y tratamientos para curar diversas enfermedades. }\end{array}$ \\
\hline Verde & Destinada a medios de calidad concernientes al tratamiento agrónomo; plantas y animales \\
\hline
\end{tabular}




\section{Metodología}

Bajo la revisión del documento de Valles (2003, P.52), en este estudio se da una propuesta de teoría emergente, enmarcada por un diseño metodológico flexible, que permite desde problemas fundados, una serie revisiones teóricas de manera sistemática, un campo de reflexión e interpretación bajo un enfoque argumentativo y propositivo, analizando el desarrollo de los conceptos y las afirmaciones teóricas a partir de perspectivas epistemológicas, ontológicas y metodológicas (Valles, 2003).

En ese sentido, los estudios realizados en el grupo de investigación GECS, sustentados por la línea de investigación en relación con la calidad en los procesos de formación en educación superior, permitieron el análisis de diversas literaturas convergentes en la reflexión referente hacia la profesionalización, desde una prospectiva que engloba una formación y caracterización de la realidad en un sentido natural de manera cualitativa; en análisis de diálogos evidenciados y contrastados en la Maestría de Educación del departamento de educación en la Universidad Militar Nueva Granda.

El proceso metodológico a partir de la teoría emergente, consistió en la construcción de un cuerpo teórico a la luz de una interpretación y el contraste dado por la perspectiva educativa y formativa; con participación de expertos, los cuales opinaron sobre el desarrollo biotecnológico y el empoderamiento que pudiese existir para una apropiación y concienciación disciplinar desde el ámbito universitario. Por tal razón, de manera continua, se describen las fases que se tuvieron en cuenta para la construcción del cuerpo teórico y la convalidación con juicio de expertos.

Primera Fase: Se constituyo un banco de documentos primarios, con cerca de 50 documentos relacionados con las categorías principales, que en primer instancia fueron la biotecnología y su aplicación en la educación superior; el empoderamiento, retomando la participación de la comunidad y el papel que tiene la mujer en la consolidación de la biotecnologías y, por último, la antelación tecnológica como objeto de conciliación, cuya perspectiva en el estudio es de conocimiento más no de artificio.

Segunda Fase: Luego de la construcción del respectivo cuerpo teórico, con acopio de subcategorías y dimensiones de análisis e interpretación, se dio cuenta de una serie de interrogantes que fueron eje principal para la estructuración de los cuestionamientos retomados en los resultados del presente estudio.

Tercera Fase: Los interrogantes fueron extrapolados desde una mirada académica y la experticia de docentes de la Universidad Militar Nueva Granada, en la facultad de Educación y Humanidades (Cuatro profesores de la Maestría en Educación), quienes dieron su punto de vista hacia la biotecnología y el papel que juega la comunidad para entender el fenómeno tecnológico y la problemáticas subyacentes del desarrollo tecnológico en el País.

Cuarta Fase: Con base en una triangulación de datos, se compararon las respuestas de los docentes junto con los apartados descritos en las categorías de análisis, llegando a la consolidación de resultados que se plantean a manera reflexiva, y a la vez, diagnóstica, para una posterior evolución del estudio.

\section{Resultados}

A través de esta reflexión que tiene como finalidad mostrar un panorama sobre el cual ya muchos autores han hecho mención por medio de diversos estudios, se establece un sin numero de conjeturas que llevaran al lector a indagar la pertinencia de la Biotecnología como profesión que enmarcar una serie de connotaciones científicas, tecnológicas, axiológicas y críticas para llegar a entender las nuevas necesidades y problemáticas que afronta el ser social en un mundo coordinado por sofisticaciones, redes informativas de 
largo alcance sustentadas en la "aldea global" (Forero, 2005).

A continuación como parte de ese ejercicio reflexivo se presentan una serie de preguntas orientadoras con el fin de dar a conocer desde una indagación, los acercamientos encontrados en el presente estudio por dar respuesta a la pertinencia de la biotecnología desde el empoderamiento como acción transformadora en instancias relacionadas con el plano académico, productivo e investigativo.

\section{¿Existe empoderamiento cuando se habla de biotecnología?}

El empoderamiento es una instancia en la cual un colectivo converge en una acción justificable en miras de conseguir un objetivo común, así pues, y desde lo descrito en la conceptualización del empoderamiento, la biotecnología responde en cierto modo a esas características desde una mirada flexible, si bien no lleva a un grupo específico en correspondencia a un bien que impacte directamente su calidad de vida, conlleva a varios sectores a interactuar de manera transversal para aprovechar al máximo recursos y procesos tecnológicos tratando de impactar y sostener la calidad de vida bajo condiciones de mortalidad, demográficas, climáticas, entre otras para regular la interacción de la sociedad con el medio ambiente.

La Biotecnología logra relacionar un empoderamiento basado en las condiciones establecidas por Engler (2005), donde el esfuerzo y la organización estructural busca suplir una necesidad considerable, en este caso la agrupación viene dada por una serie de académicos especialistas en biología, química, ingeniería, microbiología, entre muchos más, quienes por medio de saberes particulares e investigaciones concretadas en "objetos tecnológicos" (Buch, 1999) tratan de converger en un marco disciplinar, la indagación y pertinente mitigación de aspectos que hoy en día pueden tener impacto en la calidad de vida del hombre. Las cartas están abiertas y el debate sigue latente mientras opositores aluden a la improbabilidad e inmoralidad, muchos otros ven una oportunidad de transformación socio-cultural.

\section{¿Cuál es el aporte de la Bioética en el desarrollo biotecnológico como profesión?}

Es un arma de doble filo comprometer la Bioética con la biotecnología y aunque su prefijo pudiese colaborar para entablar una relación directa, la bioética a pesar de que no es una disciplina nueva está en una construcción constante desde el desarrollo humano (Rascio, 2007), su marco teórico resulta contemporáneo ante los esfuerzos de muchos humanistas y expertos en el tema en dictaminar su estatuto epistémico. Por lo tanto, la asociación con una prospectiva Biotecnológica consolidada como profesión o marco disciplinar de orden Multidisciplinar (García, 2006), puede traer consigo un mar de incertidumbres sobre la relevancia de pensar en la ética y la conciencia anidadas en la investigación, referente al aprovechamiento de nuevas tecnologías desde una base científica articulada al estudio de organismos y ambientes, bajo una finalidad de aprovechamiento en diversos campos donde el hombre hace su intervención.

Sin embargo los hechos mencionados por Carullo (2002) acerca de los constantes temores, cuestionamientos y desconocimiento alrededor de la biotecnología en especial a nivel latinoamericano, se reflejan en la importancia de tomar un postura sobre la base de una información pertinente con el fin de tomar a nivel crítico una acción de validación, con respecto a su aporte o a los juicios y normativas que afectan o limitan de forma positiva o negativa la concordancia de una Biotecnología del futuro.

\section{¿Es pertinente hablar de la Biotecno- logía como profesión del futuro?}

Se cree que la biotecnología de forma analógica al igual que la tecnología, son cuestiones que surgen en épocas contemporáneas, por el contrario han existido en diferentes etapas y coyunturas del ser, 
bajo otras denominaciones y desde ciertas limitaciones pragmáticas. Por ende aunque en el presente estudio se hable de la Biotecnología como profesión prospectiva, su práctica ha trascendido gracias a estudios que han llevado al hombre a pensar sobre la relevancia de analizar esas practicas contiguas y revaluarlas conforme el apoyo de nuevos procederes tecnológicos y científicos, el los que el hombre busca entender la magnitud de reflexionar sobre inconsistencias de orden ambiental y social en la transformación del entorno.

Autores como Muñoz (2001) realizan una puesta en escena de la biotecnologías desde referentes históricos que datan de hasta 10. 000 años atrás, cuando el hombre empezaba a cultivar y generar una domesticación de otras especies, hasta en nuestros tiempos que ya se habla de Biotecnología moderna (Muñoz, 2007) donde países denominados potencias mundiales afianzan cada vez más programas de apoyo e inversión relacionados con impacto ambiental y nuevas formas de aprovechar los recursos con uso de microorganismos, moléculas y enzimas dadas desde la misma naturaleza. A continuación a modo de infograma se presenta una interpretación de una serie de datos presentados por Carullo (2002) en determinadas áreas de aplicación de la biotecnología:

\section{Opinión en Norte América}

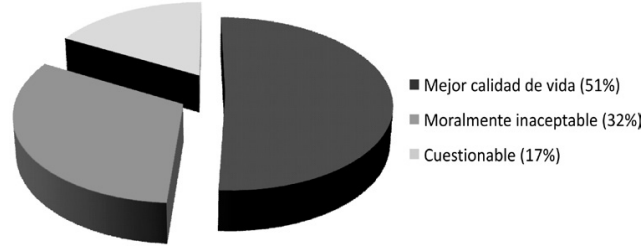

Figura 4. Estudio: concepción de uso de biotecnología realizado en el año de 1987, Tomada y reformada de Carullo (2002)

En Europa la indagación se dio particularmente y no siendo el único trabajo, bajo un estudio denominado el Eurobarometro en 1992 (Carullo, 2002), donde se aplicaron entrevistas a delegados participantes de diversos países para determinar el interés por nuevas tecnologías relacionadas con el campo científico, teniendo al respecto lo relacionado con biotecnología.

¿Qué papel toma la educación de orden superior en la consolidación de la biotecnología como profesión desde la supervivencia humana?

En relación a la motivación que llevo a generar este estudio con base en una Contribución de los Saber Pro (ECAES) desde la calidad educativa a partir de una transformación, sobresale la inquietud con respecto a las implicaciones que puede tener nuevos estudios y enfoques investigativos que den luz a nuevos prospectos de profesionales íntegros, competitivos desde estándares de calidad estipulados por la demanda productiva de los sectores tanto públicos como privados. En ese orden de ideas aquel profesional que tenga dominio de múltiples competencias y además de ello, obtenga una formación basada en principios de conciencia sobre los fenómenos culturales que engloban las actitudes, comportamientos, tradiciones y maneras de interactuar con los demás y el entorno, podrá entonces establecer una formación en función creativa y propositiva para afianzar su desempeño hacia la innovación e invención respetando los códigos y normas generados para el cuidado de la naturaleza y el medio ambiente (Clementes, 2000).

La creciente y acelerada revolución tecnológica e informática hacer que muchos ciclos y procesos incluyendo los educativos se aceleren de una forma exponencial, tanto que podemos distinguir en el caso particular de la Biotecnología el hecho de incentivar en la Universidad nacional un grupo de Bio-educación (Universidad Nacional de Colombia, 2007), en la cual los objetivos surgen del impacto de este campo en la educación básica, media y superior, tratando de establecer enfoques pedagógicos, promover cambios culturales en comunidades a través del empoderamiento de los procesos y recursos 
y además el de proponer currículos para diversos colegios de la capital de Bogotá. Así varias instituciones están realizando esfuerzos para determinar documentos maestros y propuestas que giren entorno, no sólo de la Tecnología aplicada a los procesos biológicos y químicos sino también a la par de buscar una conciencia del uso de estos medios naturales en acuerdo a sistemas sostenibles y de bajo impacto en ecosistemas y ámbitos forestales.

\section{Conclusiones}

Los constantes avances entre relaciones dialógicas de ciencia y tecnología se dan a diario, sin embargo un gran número de población mundial ignora sus beneficios o afecciones a la vida cotidiana. Ante esto surge la necesidad de trasformar la sociedad hacia un compromiso con las políticas públicas que estén vinculadas con dichos temas. Esto con el fin de crear espacios participativos en los cuales se discutan las formas en que deben ser usados todos los sistemas tecnológicos en pro del beneficio de la vida del hombre y su entorno natural.

Al hablar de vida, aterrizamos específicamente al campo de la biotecnología y sus aportes a la solución de problemas relacionados con la salud, el medio ambiente, la alimentación y todo lo correspondiente al mejoramiento de la calidad de vida del hombre. Debido a que la avasallante carrera tecnológica y su comprensión en espacios no académicos, posee aún falencias en contraste con su formación académica y profesional, en la cual debe estar presente un esquema ético que los conduzca a quehacer de su conocimiento, una posibilidad de derogar cosmovisiones absolutistas y dar paso a la garantía de salubridad equitativa para todos.

Dichas desavenencias profesionales y académicas, pueden subsistir debido a que no hay una concepción interdisciplinar y multidisciplinar fortuita de la biotecnología en el contexto, sin embargo, son grandes los esfuerzos que se hacen en el plano educativo; sobre todo en la parte biológica, médica y química, por afrontar el reto de una implementación biotecnológica innovadora que pueda dar respuesta a problemáticas complejas, como las epidemias, los cambios climáticos, el desarrollo de medicinas y la implantación y preservación de componentes naturales para fomentar la biodiversidad en el país.

Aquí la educación cumple un papel importante, pues ella desde su discurso de formación humana puede propiciar espacios de empoderamiento, que permita a toda la comunidad tecnológica construir un criterio fundamental que dirija sus esfuerzos a cumplir políticas globales referentes a la biotecnología, bioética, bioseguridad, entre otros aspectos. Trasformando en conjunto con otras áreas del conocimiento la calidad y prolongación de la vida en espacios donde la mortandad y las situaciones del diario subsistir son precarias e infrahumanas.

Para llegar allí la educación debe diseñar contenidos y espacios curriculares que desarrollen una lógica de la biotecnología tanto en estudiantes como en docentes. Todo esto acompañado de proyectos de orden tecnológico, que afiancen un pensamiento creativo sus ideas, resultado póstumo de un nivel de experticia para analizar y leer el contexto; las necesidades y problemas circundantes a nivel nacional e internacional. Para ser complementados con otras experiencias y así avanzar en la concientización de la importancia de la calidad de vida desde las invenciones tecnológicas.

Si la educación crea conciencia de la importancia de la tecnología en la vida del hombre y lleva a que la comunidad haga valer las políticas referente a esas áreas o cree espacio que modifiquen entornos en los que la tecnología no está siendo bien utilizada, se puede hablar de un empoderamiento del hombre y su sociedad al poder no solo al cambiar la conciencia colectiva, sino de avanzar en 
pro de cambiar la conciencia global que en ocasiones impone el uso de lo tecnológico y no asegura un bienestar para el hombre y su dignificación de vida.

De ahí la importancia de de formar al profesional de tecnología, el médico, el biólogo, el químico, el ingeniero, al educador, entre otros, en un empoderamiento que sustente una concepción bioética al garantizar que la biotecnología no llegue a ser manipulada, para fines nocivos y monopólicos. Como es el caso de la genética que ha hecho del hombre un objeto de estudio sin tener en cuenta sus partes sensitiva y racional que él posee y que trata de desvanecerse; se pierden junto con su parte humana en pro de un descubrimiento que nos lleve al elixir de la vida eterna.

\section{Recomendaciones}

Este estudio se abre en principio, como una diferencial de reflexión que permita poner a consideración la importancia de la proliferación de estudios específicos y particulares, en el desarrollo interdisciplinar sobre la condición biotecnológica del país. Particularmente, el estudio de investigación adelantado por el grupo GECS, da cuenta de manera general, de una concepción y ciertas aplicaciones de la biotecnología en diversos sectores productivos, no obstante el objetivo de la investigación confiere una temática social, participativa y dialógica que contribuya no solo a la comunidad educativa, sino en general, a la sociedad para que tome conciencia del impacto tecnológico. Entonces el principal objetivo estará enmarcado en apropiar una cultura de empoderamiento, asimilación y reconstrucción de aportes sustanciales para la conservación de lo natural.

En esa medida, el estudio grosso modo, incurre en los siguientes aspectos a manera de recomendaciones:

- Trabajo mancomunado de grupos relacionados con la educación, inge- niería, el derecho, la medicina y la bioquímica, para abordar problemáticas de orden complejo, supeditadas en el análisis del contexto e inferencia de problemáticas no solo ambientales y de salubridad, también las que aquejan circunstancialmente los sectores rurales y periféricos del país.

- Lograr una conceptualización biotecnológica en apego de las corrientes bioéticas que proporcionen una convergencia social y educativa, teniendo en cuenta una prospectiva históricosocial, encaminada al arraigo cultural y la participación ciudadana.

- Ofrecer como resultado de investigaciones, diversos eventos; entre ellos: coloquios, seminarios, mesas de discusión. A manera de socialización, para debatir aspectos críticos acerca del impacto tecnológico y las medidas que se pueden tomar para el desarrollo de soluciones pertinentes.

- Independientemente de la carrera formativa en el plano profesional y de posgrados, como incorporación electiva, se hace justificable la incursión de materias y cursos extensivos que manejen la biotecnología y los principios conceptuales, para dar cuenta de una socialización de los aspectos que inquieren en el desarrollo tecnológico y el uso adecuado de los recursos naturales, en pro de la preservación vitalicia de la vida en toda su extensión y el equilibrio ecológico.

- Establecer una relación más estrecha entre organismos administrativos, gubernamentales, productivos y educativos, afín de comprender desde una macro-perspectiva, el papel que desempeña cada una de estas instancias y dependencias, hacia la consolidación de una conciencia biotecnológica, que en principio, reside como una transformación cultural en las instituciones educativas, pero que trasciende a gran escala en cada uno de los sectores ya mencionados. 


\section{Bibliografía}

Aldana, Aura Violeta. (2002.) El empoderamiento femenino como acción ciudadana. En: López, M. (CIELAC), Modernización del Estado y Actores Sociales. Conferencia Centroamericana y del Caribe, Nicaragua.

Andrade, Edgar. (1996). Ambientes de aprendizaje para la educación en tecnología, en Revista educación y Tecnología, 1 (1), Pp. $21-28$.

Arias, Salvador. (1994). Campos y perspectivas de la Biotecnología: una estrategia para la introducción en el istmo centroamericano. Panamá: CADESA.

Avalos, Ignacio. (1990). Biotecnología e Industria, un ensayo de la Interpretación Teórica. San José: IICA.

Batliwala, Srilatha. (1997). El significado del empoderamiento de las mujeres: Nuevos conceptos desde la acción. En Leon, M. (Ed.), Poder y empoderamiento de las mujeres. Pp. 187-211. Bogotá: Tercer mundo editores.

Buch, Tomas. (2009). El Tecnoscopio. Buenos Aires: Aique.

Buch, Tomas. (1999). Sistemas Tecnológicos: Una contribución a una teoría general de la artificialidad. Buenos Aires: Aique.

Carullo, Juan Carlos. (2002). La percepción pública de la ciencia: el caso de la biotecnología. Universidad Nacional de Quilmes (IEC-UNQ). Buenos Aires: Biolac.

Clementes, Richard. (2000). Guía completa de las normas ISO 1400. Barcelona: Ediciones Gestión S.A.

Crespo, Patricio., De Rham Philippe., Gonzáles Glenda., Iturralde Pablo., Jaramillo Byron., Mancero Lorena., et al. (2007). Empoderamiento: conceptos y orientaciones. Quito: ASOCAM.

Duque, Juan Pablo. (2010). Biotecnología: Panorámica de un sector. España: Netbiblio, S.L.

Engler, Tomás. (2005). El empoderamiento de adultos mayores organizados en la búsqueda de un nuevo contrato social: experiencias del Banco Interamericano de Desarrollo y la Red Tiempos, en Revista Panamericana de Salud Pública, (17) 5/6. Madrid: Programa de Publicaciones de la Organización Panamericana de Salud. Pp. 438-443.
Ferrero, Andrea. (2007). La ética y Deontología profesional en la formación universitaria. En R. Sanz y A. Medina (Ed.), Bioética de la Universidad pp. 36-48. San Luis: Nueva Editorial Universitaria.

Forero, Clemente. (2005). Baldíos y cercamientos en la aldea global de la ciencia. Universidad de los Andes, en Revista de estudios sociales No. 22. pp. 81-87.

García, Francisco. (2006). "Concepto de Bioética y corrientes actuales" [En línea]. Bioética en la red. Pp. 1-9. En: http://www. bioeticaweb.com. Consultado el 14 de abril de 2011.

González, Diana. (2008). Introducción al Desarrollo Tecnológico en Construcción. Especialización en Desarrollo Tecnológico de la Construcción. San Luis: IDEC.

Intrac. (1999). Seguimiento y evaluación del empoderamiento. Documento de consulta. Inglaterra: Oxford.

Jeffé, Walter. (1992). Biotecnología y Bioseguridad en el Contexto del Mundo en Desarrollo: una Perspectiva Caribeña y Latinoamericana. En Jaffé, M. y Zaldívar, M. (Ed.), La regulación de la Biotecnología con énfasis en la liberación al medio ambiente de organismos modificados genéticamente. Pp. 237-258. San José: IICA.

Kabeer, Naila. (1997). Empoderamiento desde abajo: ¿Qué podemos aprender de las organizaciones de base?. En León, M. (Ed.), Poder y empoderamiento de las mujeres. Pp. 119-146. Bogotá: Tercer mundo editores.

Kuhn, Thomas. (1971). La estructura de las revoluciones científicas. México, D.F.: Editorial: Fondo de cultura económica.

León, Magdalena. (1997). Poder y empoderamiento de las mujeres. Bogotá: Tercer mundo editores.

Muñoz, Emilio. (2001). Biotecnología y sociedad: Encuentros y desencuentros. Madrid: Cambridge University press.

Muñoz, María Antonia. (2007). Biotecnología. Buenos Aires: Universidad Nacional De Quilmes.

Orozco, Luis y Olaya, Doris. (2005). Indicadores del Programa Nacional de Biotecnología. Bogotá: Observatorio Colombiano de Ciencia y Tecnología. 
Quintero, Rodolfo y Solórzano, Ana. (1989). Prospectiva de la Biotecnología en Latinoamética. Balance y Limitaciones. En L. Corona (Coord.), Prospectiva científica y tecnológica en América Latina. pp. 187-202. México, D.F.: UNAM.

Rascio, María. (2007). Algunas consideraciones acerca del rol de la comunidad del conocimiento en la construcción de la bioética, y su posible aporte a un desarrollo equitativo. En R. Sanz y A. Medina (Ed.), Bioética de la Universidad. pp. 19-35. San Luis: Nueva Editorial Universitaria.
Stromquist, Nelly. (1997). La búsqueda del empoderamiento: ¿en qué puede contribuir el campo de la educación?. En León, M. (Ed.), Poder y empoderamiento de las mujeres. Bogotá: Tercer mundo editores.

Universidad Nacional de Colombia. (2007). La Biotecnología "un juguete" preferido en la educación. En Revista colombiana de biotecnología, No. 2. pp. 72-78. 